\title{
Review of Noise Removal Techniques for Fixed Valued Impulse Noise
}

\author{
Priyanka Rastogi \\ M.Tech. Scholar, (D.C.), Department of ECE \\ T.I.E.I.T., Bhopal
}

\author{
Neelesh Gupta \\ Associate Professor \& HOD, Department of ECE \\ T.I.E.I.T., Bhopal
}

\begin{abstract}
Noise removal is one of the biggest challenges in the field of image processing. Impulse noise removal is one of the most necessary and important preprocessing step in digital image processing. Several median based techniques are reported in literature for different noise models. Each of them has their advantages and limitations. Most of the filters are good at noise suppression but their performance decreases in terms of edge preservation. In the review paper, various algorithms for removal of fixed valued impulse noise and their performance under different noise conditions and for various fixed valued noise models is discussed. All the techniques have their advantages and limitations. The comparative study helps in identification of most efficient algorithms in terms of edge conservation and noise suppression to restore the original image to the best possible extent given the degraded version. Most of the techniques are suitable for some particular noise models and thus does not perform satisfactorily on other types of noise models.
\end{abstract}

\section{Keywords}

Switching median filter; Image denoising; Impulse noise detection; nonlinear filter

\section{INTRODUCTION}

Image processing is widely used in all fields like atronomy, space exploration, medical imaging, scanning techniques and many others. The information carried by digital images gets corrupted by impulse noise during their acquisition or transmission [1]. Several median based techniques are studied extensively due to ease of implementation and efficiency in terms of noise removal and edge preservation as compared to their linear counterparts which cause image blurring. Simple median filter replaces each pixel with the median value, regardless of the fact whether it is corrupted or not. The uncorrupted pixels which gets filtered in turns results in loss of information and hence image quality degradation. This problem is overcome by incorporating a switching approach prior to filtering that ensures only corrupted pixels undergo filtering and uncorrupted remains intact. By modifying a switching median filter, a number of algorithms are proposed which performs better than the basic switching median filter at high noise density and various noise models. Adaptive switching median filter (AMF), boundary discriminative noise detection (BDND), center weighted median filter (CWF), progressive switching median filters (PSMF) and various other techniques are proposed for detection of impulse noise.

\section{LITERATURE REVIEW}

A lot of work has been done for the removal of fixed valued impulse noise from images. The median filter and its variants are used extensively for noise removal. Some of the significant works reported in literature is discussed.
Pei-Eng $\mathrm{Ng}$ and Kai-Kuan Ma [2] proposed a boundary discriminative noise detection technique for removing impulse noise. The filtration process consists of two stages namely impulse detection to find the processing pixel as corrupted or uncorrupted and then filtration of the corrupted pixel as the second step. The proposed algorithm is applied to each pixel of noisy image to identify whether it is corrupted or not.

In order to determine whether the current pixel is corrupted or not, this algorithm first classifies the pixels of the processing window as lower intensity impulse noise, uncorrupted pixels, higher intensity impulse noise. The pixel is considered as uncorrupted pixel if it belongs to uncorrupted pixel group. If it does not belong to uncorrupted pixel group then it is assumed to be noise and filtration process takes place. The success of this algorithm is mainly due to two boundaries that distinguish these three groups. This filtration technique is very useful for denoising heavily corrupted images. Four noise models are considered for performance evaluation. But this technique does not work on noise model 5 (NM5). Also large window size leads to more computational time.

Zhou Wang and David Zhang [3] proposed a scheme called progressive switching median filter. This algorithm is used to restore images which are corrupted by salt \& pepper noise. This method also comprises of two steps of impulse detection and then filtration of only the corrupted pixels. In this technique the impulse detector and the noise filter are applied progressively in iterative manners. The noisy pixels processed in the current iteration are utilized to help in processing of the other pixels in the subsequent iterations. The main advantage of this technique is that some impulse noise which is located in the middle of large noise blotches is detected and thus it can be filtered. This filter shows significant result at very high noise densities. This filter is used to remove salt $\&$ pepper noise only. It does not work on other four noise models.

Ashwin Thakur, Vipul Agrawal, Yogeshwar Khandagre, Alok Duby [4] proposed an approach for removal of high density salt and pepper noise through modified decision based asymmetric trimmed median filter. As all other methods available for noise removal, this process also first checks the pixel for corrupted or uncorrupted and then performs filtration process on the corrupted pixels and uncorrupted pixels are kept intact. In this technique the pixel under consideration is checked for corrupted or uncorrupted. If the value of the pixel lies between maximum and minimum gray value then it is considered as noise free pixels. On contrary, if the value of pixel is maximum or minimum gray value then it is treated as noisy pixel and hence filtered by proposed scheme. If the current pixel is 0 or 255 and all other pixels in the scanning window also have value as 0 and 255 , then the current pixel is replaced by mean of the selected window. If the window contains elements apart from 0's and 255's then 0's and 255's are eliminated and median is computed of the remaining 
pixels and current pixel is replaced by that median value. This algorithm performs satisfactorily for removing low and medium density salt and pepper noise. This filter cannot remove noise from images which are corrupted by other noise models.

Suman Shrestha [5] proposed adaptive decision based median filtering algorithm which is the combination of adaptive median filter and decision based filter. In this technique, if the median is an impulse then window size is increased by 2 in both horizontal and vertical directions and adaptive median algorithm is repeated to find out if the median is again an impulse value. If it is again an impulse then the algorithm is repeated such a number of times unless window reaches its maximum value otherwise pixel value is replaced by left neighborhood value as it is done for decision based algorithm. This technique outperforms other existing techniques at high noise density as it prevents image blurring whereas at low noise density many other techniques shows significant improvement. Noise from images which are corrupted by salt \& pepper noise can only be removed. This algorithm is not suitable for other noise models.

Raymond H. Chang, Chung-Wa Ho, and Mila Nikolova [6] proposed a scheme in which filtering of noisy pixel is done adaptive median filter and next step is to restore image using some special regularization method to preserve edge details. This method performs similar to other detection techniques at low noise density but when the noise density is high, then this technique proves to be more effective because high noise level leads to the formation of noise patches and they may be considered as noise free pixels, this reduces the performance of noise detection algorithm whereas this technique provides better results due to the high accuracy of detection technique and edge preserving property. Salt and pepper noise with high noise density are corrected with the implementation of this algorithm. But this technique does not perform for removal of all other noise models reported in literature.

K. S. Srinivasan and D. Ebenezer [7] proposed a decision based algorithm (DBA) for removal of impulse noise in which a 3 by 3 window is taken for processing. If the current pixel under consideration has value either 0 or 255 then only it is processed otherwise it is kept unchanged. But this algorithm produces streaking effects due to the replacement with neighboring pixels. This algorithm is also implemented for removal of salt and pepper noise (i.e NM1) only. Other types of noise cannot be removed with this filter.

K.Aiswarya ,V.Jayaraj and D.Ebenezer [8] proposed another technique called a new and efficient algorithm for the removal of high density salt and pepper noise from images and videos (DBUTM) which is the modification of decision based algorithm in which if a window contains elements of intensity 0 and 255 then those values are eliminated and median is calculated for the remaining values. This filter gives better results than DBA because impulses are not considered. But this technique is also useful for removing salt and pepper noise only.

\section{NOISE MODELS}

There are five types of noise models reported in the literature. They are as follows:-

1) Noise Model 1: In this noise model, the pixels are corrupted by two fixed extreme values, 0 (minimum) and 255 (maximum) with equal probability.
2) Noise Model 2: In this model, pixels are corrupted with either "pepper" noise (i.e. 0) or "salt" noise (i.e. 255) with unequal probabilities.

3) Noise Model 3: Instead of two fixed values, impulse noise takes two fixed ranges that appear at both ends with a length of $m$ each. If $m$ is 10 , noise will equal likely be any values in the range $[0,9]$ or $[246,255]$.

4) Noise Model 4: Noise model 4 is similar to noise model 3, but the probability of occurrence of these ranges in image is unequal.

5) Noise Model 5: In this model, noise takes four equally separated intensity levels. The probability of noise taking all these four values is equal. Here the gray values of noise are $15,25,225$ and 250 .

Impulse noise gets added to any image in the form of above mentioned models.

\section{DENOISING OF IMAGES}

Denoising of any image means suppression of effect of noise so that the resultant image becomes acceptable. There are two basic approaches for denoising of images. Spatial filtering methods and transform domain filtering methods. Spatial domain techniques work directly on image pixels whereas transform domain techniques are based on modifying Fourier or wavelet transform of image. Several denoising techniques are available for removal of fixed valued impulse noise due to their efficiency in noise reduction and ease of implementation. Standard median filter (SMF) is widely used due to its simplicity and noise removing capability. But this filter proves to be effective at low noise density because as noise density increases the edges of the image are not preserved [9]. This drawback was overcome by the introduction of several other techniques for the removal of salt and pepper noise. In center weighted median filter (CWM) [10], the center pixel is assigned a particular weight; this means that center pixel is repeated a number of times which is defined as a weight and then it is processed. CWM preserves more details at the expense of less noise suppression. Adaptive median filter (AMF) technique uses the approach in which window size changes adaptively for the removal of impulse noise. But this technique at high noise density leads to blurring of images due to large window size [15]. Then switching mechanism was incorporated in the filtering process so that only corrupted pixels gets filtered and uncorrupted pixels remains intact. These are called switching median filters. The performance of these filters depends on the accuracy of impulse detection schemes. The filtration scheme with min-max window [16] compares the pixel under processing window with the brightest and darkest pixels in the window and utilizes the difference value to determine the current pixel as corrupted or uncorrupted. The max filter which represents 100th percentile is used to find the brightest point in the image. The min filter is used to find the darkest point in the image. Threshold based switching median filter utilizes a suitable threshold for detection of impulse noise. Many other techniques are also available for the removal of impulse noise.

The performance of the filtration algorithms are measured in terms of visual quality and quantitatively. The objective quantitative measure used for evaluating the image restoration performance is peak signal to noise ratio (PSNR), defined as

$$
\mathrm{PSNR}=10 \log _{10}\left(\frac{255^{2}}{M S E}\right) d B
$$


Where Mean Square Error, MSE is

$$
\operatorname{MSE}=\frac{1}{M N} \sum_{i=1}^{M} \sum_{j=1}^{N}\left(y_{i, j}-s_{i, j}\right)^{2}
$$

\section{FUTURE SCOPE OF WORK}

All the techniques available for removal of fixed valued impulse noise have their own advantages and limitations. Most of the techniques available are able to remove noise corrupted by noise model 1 and noise model 2 . Very few techniques work on noise model $3 \& 4$. Some are able to remove noise effectively from highly corrupted images while some are good for images with low noise level. Some techniques remove noise but cause loss of information. Thus denoising techniques which can restore images to best possible extent are always required. Thus attempts can be made to modify some of the existing techniques so that they can perform better on existing models and can be implemented for other noise models after modifications.

\section{EXPECTED OUTCOME}

Modifications in the current techniques or by combining various techniques will result in more effective technique for image restoration. Result can be measured by visual appreciation and quantitatively in terms of increased peak signal to noise ratio (PSNR) and low mean square error (MSE). Also less number of miss detection (MD) and false alarm (FA) shows the effectiveness of good denoising technique.

\section{CONCLUSION}

In this paper we reviewed various impulse noise removal techniques reported in the literature used for the removal of fixed valued impulse noise. Most of the techniques are result of modifications in basic switching median filter. We also discussed the types of noise models by which image gets corrupted. Some of the techniques are best suited for denoising images but compromises on edge preservation. Most of the techniques are implemented for removal of salt and pepper noise only. Some methods are good at low noise density and causes blurring of images when noise content in the image is high. Thus various algorithms for the removal of fixed value impulse noise under different noise conditions and on different noise models were studied. Most of the techniques perform satisfactorily for noise model 1 and noise model 2 but their performance decreases for other noise models, Also results varies under different noise .conditions. Some are more effective for heavily corrupted images while other filters are convincing at low noise density only. Some algorithms provide a tradeoff between noise removal and edge sustentation i.e. removes noise while compromising with the details of the image. Thus noise removal is always an active field of research with the requirements of more and more efficient techniques which can restore images corrupted by impulse noise to the best possible extent.

\section{REFERENCES}

[1] Rafael C. Gonzalez and Richard E. Woods, "Digital Image Processing", Pearson Education, Second Edition, 2005 .

[2] Pei-Eng $\mathrm{Ng}$ and Kai-Kuang Ma, Senior Member, IEEE,"A Switching Median Filter With Boundary Discriminative Noise Detection for Extremely Corrupted Images", IEEE transactions on image processing , Vol.15, N0.6, June 2006.
[3] Zhou, D Zhang," Progressive switching median filter for the removal of impulse noise from highly corrupted images", Circuits and System II, Analog and Digital Signal Processing, IEEE.

[4] Ashwin Thakur et al,"AN IMPROVED APPROACH FOR REMOVAL OF HIGH DENSITY SALT AND PEPPER NOISE THROUGH MODIFIED DECISION BASED UNSYMMETRIC TRIMMED MEDIAN FILTER", International Journal of Computer Science and Mobile Computing, Vol.4 Issue.4, April- 2015, pg. 710716.

[5] Suman Shrestha, "IMAGE DENOISING USING NEW ADAPTIVE BASED MEDIAN FILTER", Signal \& Image Processing: An International Journal (SIPIJ) Vol.5, No.4, August 2014.

[6] Raymond H. Chan, Chung-Wa Ho, and Mila Nikolova,"Salt-and-Pepper Noise Removal by MedianType Noise Detectors and Detail-Preserving Regularization",IEEE TRANSACTIONS ON IMAGE PROCESSING, VOL. 14, NO. 10, OCTOBER 2005.

[7] K. S. Srinivasan and D. Ebenezer,"A New Fast and Efficient Decision-Based Algorithm for Removal of High-Density Impulse Noises", IEEE SIGNAL PROCESSING LETTERS, VOL. 14, NO. 3, MARCH 2007.

[8] K.Aiswarya V.Jayaraj D.Ebenezer," A NEW AND EFFICIENT ALGORITHM FOR THE REMOVAL OF HIGH DENSITY SALT AND PEPPER NOISE IN IMAGES AND VIDEOS",2010 Second International Conference on Computer Modeling and Simulation.

[9] S.Zhang and M.A. Karim, "A new impulse detector for switching median filters," IEEE signal Process. Lett., vol.9, no. 11, pp.360-363, Nov. 2002.

[10] Ko SJ, Lee YH," Center weighted median filters and their applications to image enhancement", IEEE Trans Circ Sys 1991;38(9):984-93.

[11] A. Fabijanska, D Sankowski ,"Noise adaptive switching median filter for impulse noise removal from extremely corrupted images",IET image processing ,August 2011.

[12] A.K. Jain ,"Fundamentals of Digital Image Processing",Prentice Hall of India, First edition.

[13] Shi-Jinn Horng, Ling-Yuan Hsub, Tianrui Lia, Shaojie Qiaoa, Xun Gonga, Hsien-Hsin Choud. Muhammad Khurram Khane,"Using Sorted Switching Median Filter to remove high-density impulse noises",Journal of Visual Communication and Image Representation, October 2013,Elsevier.

[14] Xiaoyin Xu, Member, IEEE, Eric L. Miller, Senior Member, IEEE, Dongbin Chen, and Mansoor Sarhadi," Adaptive Two-Pass Rank Order Filter to Remove Impulse Noise in Highly Corrupted Images",IEEE TRANSACTIONS ON IMAGE PROCESSING, VOL. 13, NO. 2, FEBRUARY 2004 
[15] Fanzhi Kong, Wenbin Ma, "A Fast adaptive Mean Filtering Algorithm,", 2nd International Conference on Industrial and Information Systems, IEEE 2010.

[16] Chen PY, Lien CY, Tsai CY," An effective impulse noise detector of switching median filter using min-max working window", In: Proc. IEEE Int. Conf. Intelligent Information Hiding and Multimedia Signal processing. 2007. p.234-7.

\section{AUTHOR PROFILE}

Priyanka Rastogi received her Bachelor's degree in Electronics and Communication Engineering from Truba Institute of Engineering and Information Technology Bhopal, India in 2008.

Currently she is pursuing her master of technology in digital communication from Truba Institute of Engineering and Information Technology Bhopal, India. Her research interest is image denoising. 